\title{
Unfolding designable structures
}

\author{
Cristiano L. Dia:* and Martin Grant \\ Physics Department, Rutherford Building, \\ McGill University, 3600 rue University, \\ Montréal, Québec, H3A 2 T8 Canada
}

(Dated: November 10, 2018)

\begin{abstract}
Among an infinite number of possible folds, nature has chosen only about 1000 distinct folds to form protein structures. Theoretical studies suggest that selected folds are intrinsically more designable than others; these selected folds are unusually stable, a property called the designability principle. In this paper we use the $2 \mathrm{D}$ hydrophobic-polar lattice model to classify structures according to their designability, and Langevin dynamics to account for their time evolution. We demonstrate that, among all possible folds, the more designable ones are easier to unfold due to their large number of surface-core bonds.

PACS numbers: 87.15.-v, 87.15.Aa, 87.15.By, 87.15.He
\end{abstract}

*Electronic address: diasc@physics.mcgill.ca

†Electronic address: grant@physics.mcgill.ca 


\section{INTRODUCTION}

In the human body alone, the number of different proteins is estimated to be in the range of 50,000-100,000 and this number is even larger in the biological world. However, when classified in terms of their three dimensional structures, only 1000 families of protein folds are expected to exist [1]. These structural templates for sequences of amino acids can be explained 22, 3, 4] in terms of minimalistic models where the positions of amino acids are restricted to lattice sites and the interaction energy between residues is described by a coarse-grained model. In this minimalistic approach, structures are classified according to their designability, i.e. the number of amino acid sequences they can accommodate. While some structures are not used to describe proteins, i.e. their designability is zero, a few structures are designed by an enormous number of sequences and are, therefore, stable to amino acid mutation - a desirable and natural feature for evolution. Also, highly designable structures emerge as being thermodynamically stable [2] and having protein-like symmetry [2, $3,[5]$.

Designability has also been shown to have dynamical implications: calculations suggest 6] that sequences of amino acids that fold into highly designable structures, and are thermodynamically stable, present a faster folding kinetics than random sequences - as expected for real proteins. Another important dynamical aspect of proteins is their reaction to external force fields: in their natural environment, proteins have to cope with forces during their activities. It might be expected that the set of structures which constitute recurring protein folds react differently to forces than other folds. In this paper we confirm this expectation. We study the dependence of the phase diagram on designability and show that for any combination of temperature and shear, high-designable structures are the easiest structures to unfold. This result is a consequence of how the backbone (involving strong covalent bonds) and weak bonds are distributed in these structures.

This article is organized as follow: below we review the relation between designability, thermodynamic stability and surface-to-core bonds. Following this, the model to study

unfolding is introduced, together with the mathematical framework to characterize this process. Results are then presented, followed by a discussion. 


\section{DESIGNABILITY}

The goal of this section is to review the relation between designability and thermodynamical stability for the hydrophobic-polar (HP) model in the two-dimensional compact triangular lattice [9] which describes equilibrium structures of our protein model in the next section. In the HP model [10] a protein is considered to be a chain made of polar (P) and hydrophobic $(\mathrm{H})$ like amino acids. Hydrophobicity is the only aspect of amino acids which is taken into account since it is considered the main driving force for folding [1]]. In this coarse-grained approach the energy of a sequence folded into a structure is given by the short-range contact interaction:

$$
\mathcal{H}=\sum_{i<j} \epsilon_{i, j}\left[\delta\left(\left|\vec{r}_{i}-\vec{r}_{j}\right|-\sigma\right)-\delta_{j-1, i}\right]
$$

where $\vec{r}_{i}$ is the position of the $i$ monomer and $\sigma$ is distance between lattice sites. The first delta function allows only nearest-neighbors interaction and the second delta excludes interaction between residues which are adjacent along the backbone. The interaction energy between monomers $i$ and $j, \epsilon_{i, j}$, can assume 3 values depending on the type of monomers bounded: H-H, H-P, P-P. These values are chosen to minimize the Hamiltonian when H like amino acids are buried inside the protein and $\mathrm{P}$ like amino acids are left on the surface. Following Li et al [2], we use: $\epsilon_{H H}=-2.3, \epsilon_{H P}=-1$ and $\epsilon_{P P}=0$. These values are given in arbitrary units.

In this work, proteins are 25 amino acids long and the different structures they can assume are restricted to compact self-avoiding walks on a 5 X 5 triangular lattice - see Fig. 1(d) for an example of structure. The number of independent structures that can be formed under these conditions is 352,375 . Now, given a sequence of amino acids, each structure can be scanned for its native state - i.e. its non-degenerate ground state. Sequences with degenerate ground states are believed to be unrealistic since their native states are not well defined. These sequences are therefore ignored. For our small protein, the ground state of all its $2^{25}$ binary sequences can be computed and we count the number of sequences that fold uniquely into a structure. This number corresponds to the designability of the given structure. We find that among the 352,375 structures only 135,216 ( 38\%) are non-degenerate ground states of at least one sequence.

The distribution of designability for the 135,216 structures is given in Fig. 1(a). Compact 

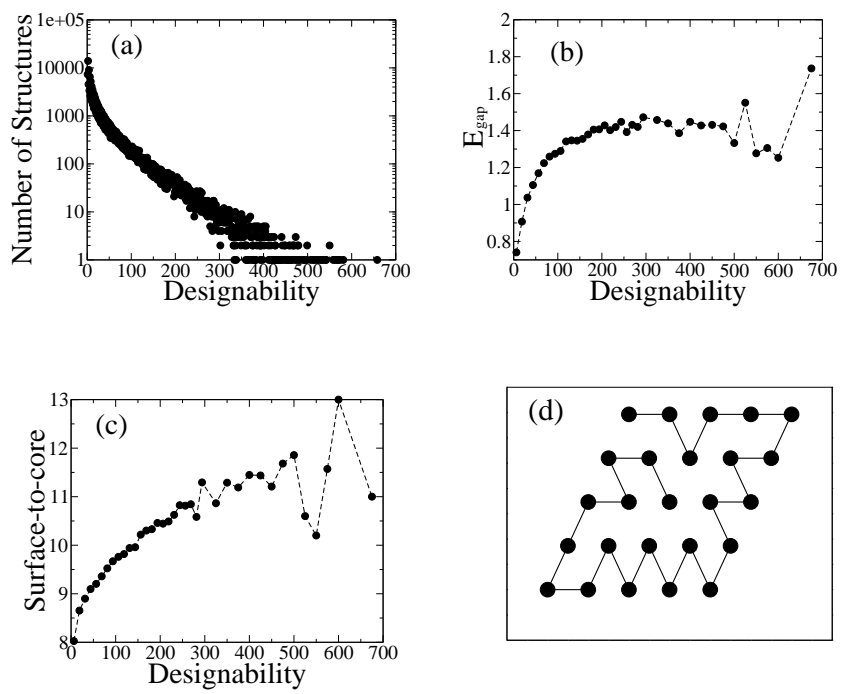

FIG. 1: a) Histogram of designability. b) Dependence of energy gap on designability. c) Number of bond connecting surface to core residues versus designability. d) Fifth most designable structure.

structures are very different when in comes to designability: many structures have a low value of designability, while just a rare number of folds accommodate more than 500 sequences. These high-designable structures are on average more stable thermodynamically than other structures. This can be shown by computing the energy difference between the ground state $E_{o}$ and the first excited state $E_{1}$ of a sequence: $E_{g a p}=E_{1}-E_{o}$. This energy difference is then averaged over sequences that have the same ground state and it is a measure of the stability of the given ground state. The correlation between $E_{g a p}$ and designability is given in Fig. 1(b) where $E_{\text {gap }}$ is averaged over a given range of designabilities.

A geometrical property of these selected structures is the large number of bonds connecting surface monomers to core monomers [5, 12, 13]. This is illustrated in Fig. 1(c) where the number of bonds connecting surface to core, averaged over structures of a given range of designability, is plotted against designability. A systematic increase of surface-to-core bonds with designability is observed. An example of structure which has a large number of bonds connecting surface to core residue is the fifth most designable structure - shown in Fig. 1(d).

\section{MODEL}

In the previous section we have shown that the surface of high-designable structures is differently connected to the core of the protein when compared to the surface of low- 
designable structures - Fig. 1(c). Therefore, since unfolding starts by unbinding surface monomers from the core, it might be expected that the dynamics of unfolding depends on designability. To investigate this idea, we present in this section a model to probe the dynamics of structures in the presence of applied forces.

In this model, the energy of each structure is accounted for by two types of potentials: monomers which are adjacent along the backbone of the protein interact through a harmonic potential otherwise the interaction is via a Lennard-Jones potential. The harmonic bond ensures that the backbone of the protein is preserved during the simulation while monomers bound by a Lennard-Jones potential can be driven apart, changing the structure of the protein. In this way, the potential energy of the chain is:

$$
V\left(r_{i, j}\right)=\sum_{i=1}^{N-1} \frac{k}{2}\left(r_{i, i+1}-\sigma\right)^{2}+\frac{1}{2} \sum_{\substack{j \neq i \pm 1 \\ j \neq i}} \epsilon\left[\left(\frac{\sigma}{r_{i, j}}\right)^{12}-2\left(\frac{\sigma}{r_{i, j}}\right)^{6}\right]
$$

in the last sum, $i$ and $j$ range from 1 to $\mathrm{N}$ and $r_{i, j}$ is the distance between monomers $i$ and $j$. $\epsilon$ and $\sigma$ are the binding energy and equilibrium length of monomers. A cut-off distance of $2.5 \sigma$ is used for the Lennard-Jones potential and $k$ is the spring constant of the harmonic potential. Notice that the model does not discriminate between $\mathrm{P}$ and $\mathrm{H}$ amino acids such that the dynamics of unfolding can be related directly to the topology of the native structure independently of amino acids sequences. Each of the 135,216 designable structures described in the previous section corresponds to a local minima of this potential energy and they can be viewed as equilibrium structures.

The fluid is modeled by including a friction and a random term $f_{i}(t)$ to the force acting on each monomer (Langevin dynamics). The intensity of the random force is given by the fluctuation-dissipation theorem. The friction force on each monomer is proportional to the relative velocity of the monomer with respect to the fluid: $-\gamma \vec{v}_{r e l}$ ( $\gamma$ is the friction coefficient). For the velocity of an element of the fluid located at position $\vec{r}$, i.e. $\vec{r}=x \hat{x}+y \hat{y}$, we use the velocity profile: $\vec{v}_{\text {fluid }}(\vec{r})=S y \hat{x}$, where $S$ is the shear rate. Inside such a fluid flow, an extended elastic object rotates and gets stretched with an intensity dependent on its orientation with respect to the fluid flow.

Putting the forces that act on a monomer together, its equation of motion inside the elongational flow is:

$$
M \frac{d^{2} \vec{r}_{i}}{d t^{2}}=\sum_{j} \vec{F}\left(r_{i j}\right)-M \gamma\left[\dot{\vec{r}}_{i}-\vec{v}_{f l u i d}(r)\right]+\vec{f}_{i}(t)
$$


where $\vec{r}_{i}$ and $\dot{\vec{r}}_{i}$ are the vectors representing the position and velocity of monomer $i$. Here, $M$ is the mass of a monomer, $\vec{F}$ is the force computed from the interacting potential. For simplicity, $\sigma, \epsilon$ and $M$ are chosen to be one. The spring is chosen to be five times stiffer than the Lennard-Jones potential: $k=5\left(72 \epsilon / \sigma^{2}\right)$. Simulations are carried out in units of the fastest atomic vibration: $\tau_{o}=2 \pi \sqrt{k / M}$; and the friction constant is given a value of: $\gamma=\left(\tau_{o} / 4\right)^{-1}$.

\section{RESULTS}

Now we quantitatively evaluate how structures with differing designabilities react to both thermal fluctuations and an applied shear force. Rather than simulate all 135,216 structures, we sample as follows. We study all the 1500 structures with highest designability, ranging from 200 to 700. For the more numerous structures which are less designable, we consider eight randomly-chosen structures for each designability. This ensemble of 3100 structures is representative of the diversity of folds.

\section{A. Shear induced unfolding}

Here we study how structures differing in designability react to an applied shear force. At zero temperature, a structure only unfolds if the shear rate is greater than $S_{c}$ - i.e. when the barrier is zero. Therefore if (at zero temperature) a structure does not unfold at a given $S_{o}$ but unfolds at $S_{o}+\delta$, the critical shear $S_{c} \equiv S_{o}+\delta / 2 . \quad \delta$ being a numerical parameter accounting for the precision of the calculation. The simulational time was 5,000 atomic vibrations. To be statistically significant we probe eight copies of each structure to different values (all differing by $\delta=0.001$ ) of the velocity flow - each copy having a different orientations with respect to the fluid. Notice that $S_{c}$ is proportional to the stability of a structure.

Results are presented in Fig. 2(a) where the dependence of $S_{c}$ on the number of times the backbone connects a surface to a core monomer is shown. Structures with a backbone zigzagging many times between surface and core are sensitive to small gradients in contrast to more linear backbone structures. In Fig. 2(b) we illustrate the dependence of $S_{c}$ on designability. In this Figure, both $S_{c}$ and designability has been averaged over structures having the same 

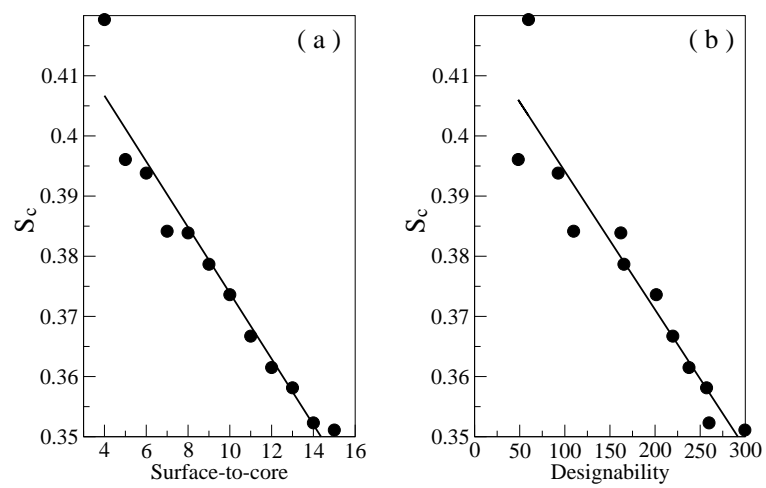

FIG. 2: (a) Dependence of the critical velocity flow on the number of surface-core bonds. (b) The dependence of $S_{c}$ on the average designability of structures having the same number of surface-core bonds. Lines in these figures are just a guide to the eye.

number of surface-to-core bonds. A clear correlation between these quantities indicates that structures which are highly designable require less shear to unfold.

\section{B. Thermal induced unfolding}

In this subsection we are concerned with thermally induced unfolding. Therefore the shear rate of our model is set to zero such that the only cause of unfolding is thermal fluctuations. We compute the unfolding time of the ensemble of 3100 structures at a temperature of 0.50 (in units of $\epsilon$ ). In our simulations, the unfolding time $\tau$ is computed by tracking the population of folded chains. The number of chains that unfold at time $t(d N / d t)$ is proportional to the population of folded chains $N(t)$. In this case, $N(t)=N_{o} \exp (-R t)$ where $R$ is the rate of unfolding and the characteristic unfolding time is given by the inverse of the rate $\tau=1 / R$. We use 1,000 copies (i.e. $N_{o}=1000$ ) of each structure in the simulations. The larger the unfolding time of a structure, the more stable it is to thermal fluctuations. In Fig. 3(a) the unfolding time is plotted versus the number of surface-tocore bonds. The clear downward trend of this figure indicates that structures with many surface-to-core bonds unfold faster. Figure 3(b) presents the correlation between unfolding time and designability. This Figure was obtained by averaging both time of unfolding and designability over structures having the same number of surface-to-core bonds. Again a 

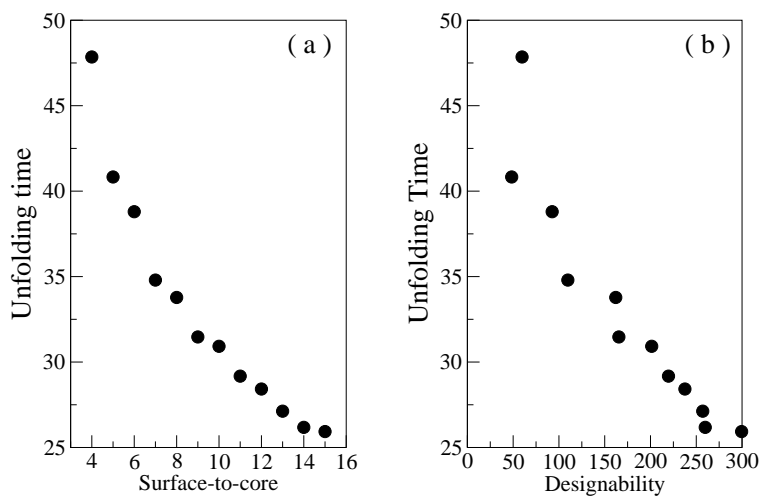

FIG. 3: Dependence of the unfolding time on (a) number of surface-to-core monomers and (b) designability.

clear correlation indicates that on average, structures with low designability are more robust to thermal fluctuations.

\section{Designability dependent phase-diagram}

We now study how the designability of a protein affects its phase-diagram. This diagram is constructed by computing the applied shear rate required to unfold a structure in 5, 000 units of time at different temperatures. This shear rate is then averaged over structures having the same number of surface-to-core bonds. Notice that the computed shear delimits two regions of the diagram: folded structures are found below this shear and unfolded structures above it. In Fig. 4 the phase-diagram is shown for structures having 4 and 15 surface-to-core bonds. These two sets of structures have an average designability of 59.71 and 299.55 respectively. At any temperature, the set of structures with lower designability is more robust and require a higher shear rate to unfold. One can therefore state that high designable structures are easier to unfold than low designable ones. 


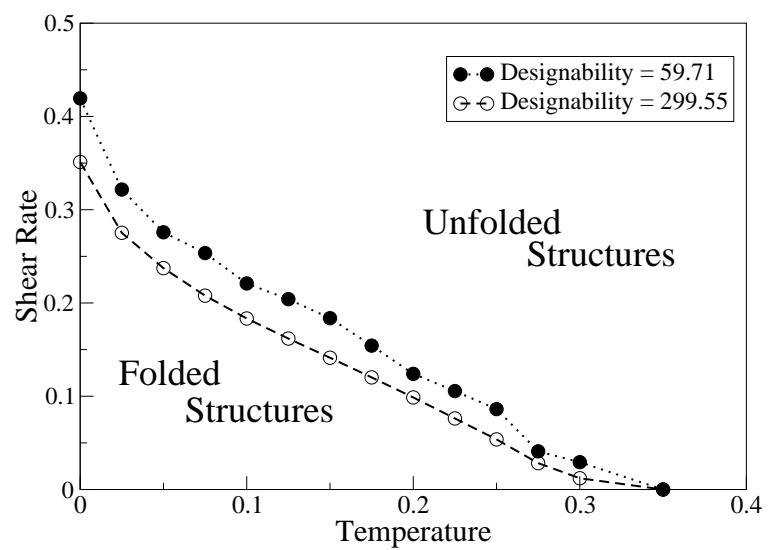

FIG. 4: Phase diagram of the set of structures having 4 and 15 surface-to-core bonds - filled and open circles, respectively. The latter (former) has an average designability of 299.55 (59.71).

\section{DISCUSSION}

The relation between thermodynamical stability and designability, called the designability principle, has been shown in Fig. 1(b): highly designable structures are more stable thermodynamically than low designable ones. In marked contrast to the designability principle, we have shown that highly designable structures are easier to unfold than low designable ones - i.e. they are weaker. The implication is that, although highly designable structures are more stable in the folded region of the phase diagram, they require less perturbation to unfold. We speculate this may be related to protein flexibility.

A qualitative explanation is as follow. We have shown that highly designable structures are weaker due to the large number of surface-to-core bonds they contain. Consequently, these structures contain many small domains (i.e. sub-structures). These are easy to unfold: only a few bonds need to rupture in order to separate the domains. In contrast, low designable structures have few surface to core bonds. As a result, many weak bonds are aligned forming domains where monomers are correlated over long distances. For those structures, the time of unfolding is dominated by the slow unbinding of the largest domain. Therefore, these low designable structures can be said to be stronger.

Also, the presence in large number of surface-to-core bonds makes it difficult to transform 
highly designable structures into other distinct compact shapes through local rearrangements of the backbone [3]. Such a transformation would require the partial unfolding of the structure, which is unlikely in the region of the phase diagram where folded structures are at equilibrium, followed by folding into the new shape. Therefore, the presence of surface-tocore bonds might explain why high designable structures are thermodynamically stable but easier to unfolding. Finally, we expect interesting insights to be obtained by expanding the model to three dimensions and including hydrodynamics effects (i.e. modeling the solvent explicitly).

[1] S. E. Brenner, C. Chothia, and T. J. Hubbard, Current Opinion in Structural Biology 7, 369 (1997).

[2] H. Li, R. Helling, C. Tang, and N. Wingreen, Science 273, 666 (1996).

[3] H. Li, C. Tang, and N. S. Wingreen, Proc. Natl. Acad. Sci. USA 95, 4987 (1998).

[4] J. Miller, C. Zeng, N. S. Wingreen, and C. Tang, PROTEINS: Structure, Function, and Genetics 47, 506 (2002).

[5] T. Wang, J. Miller, N. S. Wingreen, C. Tang, and K. A. Dill, Journal of Chemical Physics 113, $8329(2000)$.

[6] R. Melin, H. Li, N. S. Wingreen, and C. Tang, Journal of Chemical Physics 110, 1252 (1999).

[7] H. Cejtin, J. Elder, A. Gottlieb, R. Helling, H. Li, J. Philbin, N. Wingreen, and C. Tang, Journal of Chemical Physics 116, 352 (2002).

[8] H. Chen, X. Zhou, and Z.-C. Ou-Yang, Physical Review E 64, 041905 (2001).

[9] A. Irback and E. Sandelin, Journal of Chemical Physics 108, 2245 (1998).

[10] K. F. Lau and K. A. Dill, Macromolecules 22, 3986 (1989).

[11] W. Kauzmann, Adv. Protein Chem. 14, 1 (1959).

[12] R. Helling, H. Li, R. Melin, J. Miller, N. Wingreen, C. Zeng, and C. Tang, Journal of Molecular Graphics and Modelling 19, 157 (2001).

[13] C. T. Shih, Z. Y. Su, J. F. Gwan, B. L. Hao, C. H. Hsieh, J. L. Lo, and H. C. Lee, Physical Review Letters 84, 386 (2000).

[14] M. P. Allen and D. J. Tildesley, Computer Simulations of Liquids (Clarendon, Oxford, 1990).

[15] P. Hanggi, P. Talkner, and M. Borkovec, Reviews of Modern Physics 62, 251 (1990). 
[16] K. Binder, Physical Review A 29, 341 (1984).

[17] Y. Sang, M. Dubé, and M. Grant, Physical Review Letters 87, 174301 (2001).

[18] L. Gunther and B. Barbara, Physical review B 49, 3926 (1994).

[19] O. Dudko, A. Filippov, J. Klafter, and M. Urbakh, PNAS 100, 11378 (2003).

[20] C. L. Dias, M. Dube, F. Oliveira, and M. Grant, Phys. Rev. E 72, 011918 (2005). 